\title{
Advances in DIY Health and Wellbeing
}

Aisling Ann O'Kane

University College London

London, UK

a.okane@cs.ucl.ac.uk

\section{Amy Hurst}

University of Maryland

Baltimore, MA, USA

amyhurst@umbc.edu

\section{Gerrit Niezen}

Tidepool.org

San Francisco, USA

gerrit@tidepool.org

\section{Nicolai Marquardt}

University College London

London, UK

n.marquardt@ucl.ac.uk

\section{Jon Bird}

City University London

London, UK

jon.bird@city.ac.uk

\section{Gregory Abowd}

Georgia Institute of Technology

Atlanta, GA, USA

abowd@gatech.edu

\section{Abstract}

The choice of consumer healthcare and wellbeing technologies has never been greater, and the introduction of consumer wearable technologies and inexpensive sensor kits means that developing bespoke

Permission to make digital or hard copies of part or all of this work for personal or classroom use is granted without fee provided that copies are not made or distributed for profit or commercial advantage and that copies bear this notice and the full citation on the first page. Copyright for third-party components of this work must be honored. For all other uses, contact the Owner/Author. Copyright is held by the owner/author(s). CHI'16 Extended Abstracts, May 07-12, 2016, San Jose, CA, USA ACM 978-1-4503-4082

3/16/05. http://dx.doi.org/10.1145/2851581.2856467

personalized health devices is now possible. For example, there is a growing community making DIY diabetes technologies and the trend is spreading to other health areas where people want to design, customize, manufacture and disseminate their own DIY health and wellbeing technologies. Although the $\mathrm{CHI}$ community has started to investigate these trends, the pace that motivated open-source health 'makers' and 'hackers' are developing technologies means that there is a need to bring together researchers to discuss the $\mathrm{HCI}$ implications of this changing landscape.

\section{Author Keywords}

Maker culture; hackers; health; assistive technologies; wellbeing; DIY; end-user customization; open-source.

\section{ACM Classification Keywords}

H.5.m. Information interfaces and presentation (e.g., $\mathrm{HCI}$ ): Miscellaneous.

\section{Background}

The widespread availability of affordable consumer technologies, such as wearables, activity trackers, smartphone apps, and other interactive technologies, enables people to track many different aspects of their lives. There is an increasing awareness that many consumer devices have technical capabilities that would be useful for self-management or self-tracking health and wellbeing, even when they were not designed for this domain, and people are starting to appropriate and 
modify off-the-shelf products [13]. Furthermore, the availability of inexpensive and easy to use sensors, which can be combined with open-source

microcontroller platforms, has led to increasing numbers of people creating and sharing bespoke opensource healthcare and wellbeing systems that others can customize and use to build their own health technologies.

DIY and making activities, such as hacking, tinkering, repairing and crafting are growing in popularity [1], and increasing numbers of people from around the world are motivated to share their open-source designs with a larger audience, often without compensation for their time. One motivation is that they want others to also benefit from their 'hacked' technology. Spreading this 'deviant' expertise to benefit a larger group has been successful in other domains, where the 'Power of Positive Deviance' has been realized in addressing issues in child nutrition, public health, and education [6]. The success of positive deviance "rests on its ability to mobilise the community to identify role models within its midst who use uncommon, but demonstrably successful, strategies to tackle common problems" [6].

Much of the work that is being done in DIY health and wellbeing is happening in the privacy of people's homes, and much of it may be kept private because of legal issues such as the hacked devices not meeting the requirements of standards bodies [4]. However, there are a few grassroots maker communities at the

forefront of DIY health and wellbeing, such as the one designing, customizing, manufacturing technologies for Type 1 Diabetes, that are actively disseminating opensource technologies as widely as possible.
The Case of Type 1 Diabetes DIY Technologies Type 1 Diabetes (T1D) is a chronic condition that requires daily self-management of blood glucose (BG) levels by balancing exercise, diet, and injected insulin. Self-care includes calculating medication doses to inject based on factors such as food consumption and current BG levels, which is often measured with a mobile BG meter. In addition to BG meters, there are various commercially available self-care devices including wearable insulin pumps and continuous glucose monitors (CGM), mobile apps, and other digital tools that continue to come on the market. Research has shown that adults with T1D are adopting, carrying, and using devices in varied and individualized ways to suit their own everyday lives [11], and demonstrated a need and desire for bespoke and personalized self-care devices [7].

Beyond commercially available devices, there is a community of makers that are developing their own bespoke solutions to T1D needs. These makers personalize their T1D devices to varying degrees. At the lower fidelity end, they can adopt accessories such as luggage tags to carry their devices (Figure 1) or order customized stickers or skins to add to their devices. At the higher fidelity end, there are movements such as \#WeAreNotWaiting who use their technical expertise to create and release bespoke technology solutions at a faster rate than manufacturers or research labs. \#WeAreNotWaiting believe manufacturers are taking too long to release products that provide functionalities that they find important, such as being able to upload glucose meter data to the cloud. DIY groups such as Nightscout have modified CGMs to broadcast "CGM in the Cloud' and communicate with other devices that are not reachable 


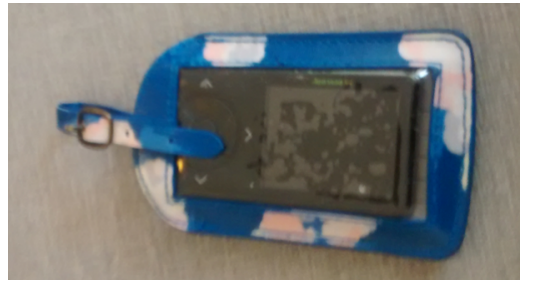

Figure 1: Luggage tag used to carry a CGM

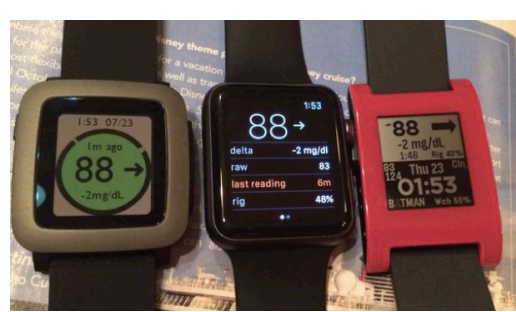

Figure 2: Three different smartwatches running Nightscout [14]

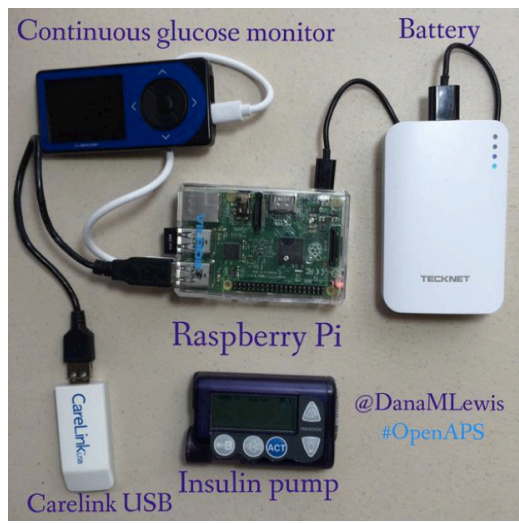

Figure 3: "What does a closed loop artificial pancreas look like?" [15] using the commercially available CGM Bluetooth capabilities. This not only enables people to broadcast their results to their smartphones and smartwatches [14], but also allows parents to monitor their children's BG levels remotely and in real time, something they couldn't do with consumer devices (Figure 2) [13, 14].

There have also been moves to use off-the-shelf CGMs and insulin pumps in conjunction with programmable devices such as Raspberry Pi to develop more advanced T1D technologies. The closed loop 'artificial pancreas,' where a glucose monitor is connected to an insulin pump and addresses glucose levels through automatic medication injection without human assistance, has not been released by any research group or manufacturer to date. However, there are already people using their own open-source DIY closed loop pancreas (Figure 3) [15].

\section{Wider HCI Research Implications}

Although there have been great strides in DIY diabetes technologies, there have also been challenges that have emerged that are important to discuss as they exemplify some of the general $\mathrm{HCI}$ issues that arise in DIY health and wellbeing. For instance, despite the availability of open-source designs, the majority of people who would benefit from personalized self-care tools do not have the relevant technical expertise to participate in this DIY maker culture [5]. For example, there are over 14,000 Facebook members of CGM in the Cloud, but under 3500 downloads of Nightscout at the time of writing. Furthermore, the development of useful tutorials for open-source technologies has been shown to be difficult in the HCI domain, even with technically inclined participants shown to struggle with replicating designs [12]. It has been argued that hacking is not practiced in a vacuum independent of political, social, or economic forces [9] and there are other barriers for people that can make them feel excluded from the maker culture, for instance, feeling intimidated about getting involved in 'hacking' [10].

Despite these barriers, the DIY making trend looks like it will continue with other groups organizing and creating more grassroots groups. This includes established makers openly sharing assistive technology on community platforms, for example, Thingiverse [2], hack-a-thons for specific conditions, maker events for health and wellbeing, and people actively wanting to start up a \#WeAreNotWaiting movement for technologies such as hearing devices [16]. There is an opportunity for HCI to support the development of the necessary skills to customize, design and manufacture bespoke health and wellbeing devices by identifying and developing support systems for end-users. These strategies and tools can be further expanded to benefit other groups with growing appetites for bespoke technologies and result in a rising research area in HCI.

Goals and Challenges for the Workshop

The workshop will bring together HCI researchers to discuss the emerging challenges in the field of DIY Health and Wellbeing, including the following issues.

DisSEMINATING DIY HEALTH TECHNOLOGIES FURTHER How can the benefit of open-source DIY bespoke technologies reach beyond the early adopters and 'hackers' to motivated individuals that may not have the necessary technical skills? How can designs be 
disseminated safely, but also customised for individuals?

FinANCIAL Motivations in DIY HEALTH AND WELLBEING 3D printing shops are becoming more widespread and cheap sensor kits are readily available - will people without the technical skills be willing to pay third parties to manufacture and build open-source technologies? How do the designers of open-source technology feel about others making a profit from designs that they have made freely available? How do people who would like to profit from their designs fit into this DIY movement? Does it matter whether designs are open-source? How do individuals with financial motivations integrate with an open source community?

SPREADING DIY KNOWLEDGE TO OTHER COMmUNITIES How can the lessons learned from one community spread to others? For instance, DIY bespoke technologies have been popular in the assistive devices domain for a few years and the \#WeAreNotWaiting diabetes movement has taken off in earnest in the last two years, and other communities are trying to learn from their success. For example, there are people trying to create an open-source movement around hearing devices, @hearinghacks [16], in order to emulate the diabetes open-source movement.

Weighing the Benefits of DiY HeALTH AND WELlbeing TECHNOLOGIES

How can people make informed decisions about getting involved in DIY making of technology versus buying devices off-the-shelf? How can we study the benefits and disadvantages of DIY devices versus off-the-shelf ones? How can cost-benefit be measured?
The workshop aims to attract a diverse group of HCI researchers who are interested in health and wellbeing and we therefore expect that further issues,

opportunities and research challenges will emerge at the workshop. As this DIY domain is still new but rapidly growing, the discussions that take place at the workshop will be decided by the participants

themselves to allow for a range of topics related to DIY health and wellbeing to be covered.

\section{Organizers}

The organizers of this workshop have experience running workshops and a varied skill set that will ensure the success of this CHI workshop.

Aisling Ann O'Kane is currently finishing a PhD and about to start a postdoctoral fellowship examining DIY maker practices among people with Type 1 Diabetes. She has been a co-organizer of two CHI workshops: in 2013 on fieldwork in healthcare HCI [3]; and in 2014 on theory in healthcare HCI [8]. She has maintained a website for these workshops and was a co-editor on two books that resulted directly from the 2013 workshop.

Amy Hurst is an Associate Professor and principal investigator on the successful NSF project on DIY opensource assistive technologies [2]. She has experience organizing workshops and editing special issues.

Gerrit Niezen is an embedded software engineer at Tidepool.org, a non-profit open-source diabetes data company. He was previously a postdoc on the CHI+MED project, where he built an open-source DIY infusion pump [11] and organized and ran an open- 
source hardware for healthcare workshop at the Things conference in Germany.

Nicolai Marquardt is a lecturer in Physical computing at $\mathrm{UCL}$, has experience running co-design workshops and prototyping sessions, and is mentoring Aisling's fellowship on DIY diabetes technology. He has experience running workshops at ACM conferences and has co-edited associated special issues.

Jon Bird is a lecturer in HCI and health informatics at City University London. He is active in maker culture, both as a builder and researcher, and has extensive experience of organizing workshops.

Gregory Abowd is a Professor and an ACM distinguished fellow. He is involved in maker projects and teaches students about these processes. He has extensive experience running workshops and editing major volumes of work.

\section{Website}

The workshop website will be launched through the website that hosted the CHI 2013 and CHI 2014 workshops on healthcare HCI.

hcihealthcarefieldwork.wordpress.com has successfully advertised two workshops and two healthcare HCI book launches. It has over 100 people signed up to get updates about the site and has had over 3000 views since it started. This website will be updated for the CHI 2016 workshop to host information before the call is sent out, and will be maintained past the date of the conference to provide updates on the progress of the special issue and other related activities, and collaborations that may result from this workshop.

\section{Pre-Workshop Plans}

The workshop will be widely advertised over numerous email lists to reach out to a variety of researchers interested in DIY health and wellbeing technologies. This includes the CHI, SIGHIT, ASSETS, and hci.fieldwork. healthcare mailing lists. The workshop call will also be disseminated widely through personal contacts and advertising on social media.

The submitted papers will be distributed for review among the program committee, all of whom are members of the $\mathrm{CHI}$ healthcare and assistive technology communities. This program committee consists of Adrian Bassone and George Buchanan of City University London, Yunan Chen of UC Irvine, Shaun Kane of University of Colorado, Dmitri Katz of Open University, Francisco Nunes of TU Vienna, Sunyoung Park of University of Michigan, Madhu Reddy of Northwestern University, John Rooksby of Glasgow University, Kate Sellen of OCAD University, Katarzyna Stawarz of UCL, Anja Thieme of Microsoft Research, and Nervo Verdezoto of Aarhus University. The final decisions about which papers will be accepted for the workshop will be based on these reviews and a meeting among the organizers, in order to ensure breadth of content and participants.

After the participants have been notified that they have been accepted to the workshop, we will ask them to participate in setting the agenda for the day. Using the tool well-sorted.org, they will be able to add topics that they would be interested in discussing, and through a collaborative online sorting activity, be able to set an agenda for discussion by sorting topics into discussion topics. This will ensure that the workshop discussions are selected through a bottom-up process and that 
participants have the chance to add topics that they feel should be addressed in the larger group.

Participants will be asked to read accepted submissions that will be posted on the website before the workshop in order to become familiar with other participants' work. They will also be asked to bring any 'hacked' health and wellbeing devices that they would like to show. Participants will be reminded to sign up for the full conference and will be sent the finalized agenda. Food allergy information will be gathered for planning lunch and dinner near the workshop venue.

\section{Workshop Structure}

09:00-09:30 Welcome - The day will be kicked off with a welcome from the organizers, an overview of the agenda, and a reminder of the goals of the workshop.

09:30-10:00 Keynote - The workshop will be started with a keynote from Brandon Arbiter from Tidepool.org, a proponent of open-source software and hardware for diabetes self-management and T1D maker. The keynote will cover the impressive strives that the diabetes 'maker' community have made.

10:00-10:30 Introductions - Following the keynote, an icebreaker will be held to get people introduced to each other, which will lead into the participant minute madness presentations.

10:30-11:00 Coffee Break - This networking break will allow participants to start conversations and ask followup questions generated by the Introductions.

11:00-12:30 Show and Tell - Participants will be able to display and demonstrate any health and wellbeing technologies that they have brought with them during informal demonstrations. This will include examples of diabetes technologies from the keynote, off-the-shelf technologies that have been 'hacked' and also demonstrations of sensor kits.

12:30-14:00 Lunch - A restaurant will be booked nearby to accommodate the participants, and informal networking between attendees will be possible.

14:00-14:30 Framing the Discussions - After lunch, the results of the well-sorted.org pre-workshop activity will be presented. At this point, the participants will be split into rotating groups based on a prearranged scheme.

14:30-15:30 Group Discussions - Small groups will discuss the subjects that came out of the well-sorted activity (for instance, "How can designs be safely disseminated?"). These discussions will culminate in hand drawn posters and brief 2-minute presentations by each group. The groups will then break up and mix with others for the next group discussions.

15:30-16:00 Coffee Break - Informal networking and continued discussions will take place.

16:00-17:00 Group Discussions - Small group discussions will continue based on the well-sorted.org topics, with posters being created and 2-minute presentations by the groups.

17:00 - 17:30 Wrap-up and discussion of plans for follow-up activities, joint publications and special issue.

17:00-19:00 Break and Dinner - The organizers will split, with some taking pictures of the posters to post 
on the website and clearing the room, and the others taking the group to a nearby pub/cafe to have informal discussions before dinner. Participants can choose to join at the pub or head back to hotels before meeting for dinner at a restaurant close to the conference venue so that networking can continue.

\section{Post-Workshop Plans}

There will be photos taken throughout the day of the event and the posters and these will be displayed on the website, given the permission of the attendees. The accepted workshop papers will also be hosted on the website and entered into UCL's repository system so that they can be cited in Google Scholar. The posters and the findings of the day will culminate into a call for a special issue journal on DIY Health and Wellbeing Technologies. The results of the day will be written up by the organizers to act as the introduction of this special issue. We are currently talking to HCI journals to host this special issue. The call will take place within one month of the workshop, and it will be open to the larger HCI community.

\section{Call for Participation}

In conjunction with $\mathrm{CHI} 2016$, a workshop will be held to discuss the rapidly growing DIY Health and Wellbeing domain. Maker and hacker culture has driven advances in diabetes technology and the development of other bespoke assistive technologies, and there is potential for growth in this field to other health and wellbeing domains, and many HCI concerns and questions are starting to arise.

A keynote by Brandon Arbiter of Tidepool.org will outline advances in DIY diabetes, and short introductions by attendees will follow along with a show-and-tell of current DIY Health and Wellbeing technologies. Group discussions will occur on topics that have been chosen and developed by attendees prior to the workshop.

Submissions are welcome on topics related to DIY health and wellbeing and $\mathrm{HCI}$, including: case studies in hacking health or wellbeing devices; reports of ongoing research on DIY health or wellbeing; reflections on the opportunities of patient-led technology projects; studies of grassroots maker communities for health and wellbeing; and, challenges and opportunities for $\mathrm{HCI}$ research in DIY heath and wellbeing.

Four-page position papers in Extended Abstract format should be submitted to Easychair by January 8th 2016 at 5pm GMT (papers submitted by December 15th 2015 will be considered for early acceptance). At least one author of each accepted position paper must attend the workshop and all participants must register for both the workshop and for at least one day of the CHI 2016 conference.

More information can be found here,

https://hcihealthcarefieldwork.wordpress.com/chi2016-diy-health-workshop/. An open call for a special issue on DIY Health and Wellbeing technologies will follow the workshop.

\section{References}

1. Jeffrey Bardzell, Shaowen Bardzell, and Austin Toombs. 2014. "Now That's Definitely a Proper Hack": Self-made Tools in Hackerspaces. Proceedings of the SIGCHI Conference on Human Factors in Computing Systems, ACM, 473-476.

http://doi.org/10.1145/2556288.2557221 
2. Erin Buehler, Stacy Branham, Abdullah Ali, et al. 2015. Sharing is Caring: Assistive Technology Designs on Thingiverse. Proceedings of the 33rd Annual ACM Conference on Human Factors in Computing Systems, ACM, 525-534. http://doi.org/10.1145/2702123.2702525

3. Dominic Furniss, Aisling Ann O'Kane, Rebecca Randell, Svetlena Taneva, Helena Mentis, and Ann Blandford. 2013. HCI fieldwork in healthcare: creating a graduate guidebook. CHI'13 Extended Abstracts on Human Factors in Computing Systems, ACM, 3203-3206.

4. Dan Hurley. 2014. Diabetes Patients Are Hacking Their Way Toward a Bionic Pancreas. Wired Magazine. Retrieved September 21, 2015 from http://www.wired.com/2014/12/diabetes-patientshacking-together-diy-bionic-pancreases/

5. Silvia Lindtner, Garnet D Hertz, and Paul Dourish. 2014. Emerging Sites of HCI Innovation: Hackerspaces Hardware Startups \& Incubators. Proceedings of the SIGCHI Conference on Human Factors in Computing Systems, ACM, 439-448. http://doi.org/10.1145/2556288.2557132

6. David R Marsh, Dirk G Schroeder, Kirk A Dearden, Jerry Sternin, and Monique Sternin. 2004. The power of positive deviance. BMJ (Clinical research ed.) 329, 7475: 1177-9. http://doi.org/10.1136/bmj.329.7475.1177

7. Aisling Ann O'Kane, Yvonne Rogers, and Ann Blandford 2015. Concealing or Revealing Mobile Medical Devices? Designing for Onstage and Offstage Presentation. Proceedings of the 33rd Annual ACM Conference on Human Factors in Computing Systems, 1689-1698.

8. Kate Sellen, Dominic Furniss, Yunan Chen, Svetlena Taneva, Aisling Ann O'Kane, and Ann Blandford. 2014. Workshop Abstract: HCI Research in Healthcare: Using Theory from Evidence to Practice. CHI '14 Extended Abstracts on Human Factors in Computing Systems, ACM, 87-90. http://doi.org/10.1145/2559206.2559240

9. Yuling Sun, Silvia Lindtner, Xianghua Ding, Tun Lu, and Ning Gu. 2015. Reliving the Past \& Making a

Harmonious Society Today: A Study of Elderly Electronic Hackers in China. Proceedings of the 18th
ACM Conference on Computer Supported Cooperative Work \& Social Computing, ACM, 44-55. http://doi.org/10.1145/2675133.2675195

10. Austin L Toombs, Shaowen Bardzell, and Jeffrey Bardzell. 2015. The Proper Care and Feeding of Hackerspaces: Care Ethics and Cultures of Making. Proceedings of the 33rd Annual ACM Conference on Human Factors in Computing Systems, ACM, 629-638. http://doi.org/10.1145/2702123.2702522

11. James Christopher Vincent, Gerrit Niezen, Ann Aisling O'Kane, and Katarzyna Stawarz. 2015. Can Standards and Regulations Keep Up With Health Technology? JMIR mHealth uHealth 3, 2: e64.

http://doi.org/10.2196/mhealth.3918

12. Ron Wakkary, Markus Lorenz Schilling, Matthew A Dalton, et al. 2015. Tutorial Authorship and Hybrid Designers: The Joy (and Frustration) of DIY Tutorials. Proceedings of the 33rd Annual ACM Conference on Human Factors in Computing Systems, ACM, 609-618. http://doi.org/10.1145/2702123.2702550

13. John Walsh, Ruth Roberts, Richard Morris, and Lutz Heinemann. 2015. Device Connectivity: The Next Big Wave in Diabetes. Journal of Diabetes Science and Technology http://doi.org/10.1177/1932296814568806

14. @WeAreNotWaiting, 2015. "One Group, Many Solutions" \#CGMintheCloud \# Nightscout \#WeAreNotWaiting \#PebbleTime \#AppleWatch \#Pebble. Tweet. (23 July 2015). Retrieved July 23 2015 from https://twitter.com/WeAreNotWaiting/ status/624155264460066816.

15. @danamlewis. 2015. "What does a closed loop artificial pancreas look like?" (The size of your battery may vary :) ) \#DIYPS \#OpenAPS. Tweet. (17 Sept, 2015) Retrieved September 17, 2015 from https://twitter.com/danamlewis/status/6447000394665 12384

16. @HearingHacks, 2015. Any of the founders of \# wearenotwaiting in London? Trying to build something similar for \# hearingaids industry and could use advice. Tweet. (17 Aug 2015). Retrieved August 17, 2015 from https://twitter.com/hearinghacks/status/63329226. 